\title{
TOLERANCIA DE SORGO PARA GRANO A DOS HERBICIDAS
}

\section{TOLERANCE OF GRAIN SORGHUM TO TWO HERBICIDES}

\author{
Enrique Rosales-Robles*, Ricardo Sánchez-de-la-Cruz y Luis A. Rodríguez-del-Bosque
}

\begin{abstract}
Campo Experimental Río Bravo, Instituto Nacional de Investigaciones Agrícolas, Forestales y Pecuarias. Km. 61 Carretera Matamoros-Reynosa. 88900, Río Bravo, Tamaulipas, México. Tel. (899) 934-1045.

${ }^{*}$ Autor para correspondencia (enrique_77840@yahoo.com)
\end{abstract}

\section{RESUMEN}

En el norte de Tamaulipas, México, la fitotoxicidad causada por los herbicidas en sorgo (Sorghum bicolor L. Moench.) es un problema común debido a las épocas y dosis de aplicación inapropiadas. Dicha fitotoxicidad afecta el crecimiento del cultivo y en ocasiones reduce su rendimiento. De 2006 al 2008 se evaluó en campo la respuesta de cuatro híbridos de sorgo ('P-82G63', 'RB-Patrón' , 'DK-54' y 'Ámbar') a la aplicación de dos herbicidas (prosulfuron y 2,4-D) en dos dosis (de etiqueta, 1X: prosulfuron $22.8 \mathrm{~g}$ i.a. ha- ${ }^{-1}$ y 2,4-D $720 \mathrm{~g}^{\text {i.a. }}$ ha $^{-1}$; $\mathrm{y}$ una sobredosis de $50 \%, 1.5 \mathrm{X}$ ) en dos etapas fenológicas (V5, 5 hojas liguladas; y V8, 8 hojas liguladas). No se encontraron diferencias entre los genotipos a los tratamientos de herbicidas, pero sí entre dosis y herbicidas. Prosulfuron a $1 \mathrm{X}$ aplicado en V5 sólo causó una fitotoxicidad leve de $6 \%$ que redujo la altura del cultivo, fitotoxicidad que subió a un máximo de $19 \%$ con la dosis $1.5 \mathrm{X}$ en la etapa V8, pero en ningún caso afectó el rendimiento. El herbicida 2,4-D aplicado en plantas en las etapas V5 y V8 con las dosis $1 \mathrm{X}$ y $1.5 \mathrm{X}$ causó una fitotoxicidad significativa de 12 a $24 \%$ y redujo el rendimiento de 12 a $16 \%$, porque provocó disminución del número de granos por panoja. Por ello debe evitarse la aplicación de 2,4-D al sorgo en estas condiciones. En sorgo, el prosulfuron es un herbicida más conveniente que el 2,4-D porque causa menor fitotoxicidad.

Palabras clave: Sorghum bicolor, tolerancia a herbicidas, altura de planta, rendimiento.

\section{SUMMARY}

In northern Tamaulipas, México, herbicide toxicity is a common problem in grain sorghum (Sorghum bicolor L. Moench.) caused by inappropriate herbicide dosages and inadequate synchronization of spraying with crop growth stages. Herbicide toxicity affects crop growth and may reduce yield. From 2006 to 2008, the response of four grain sorghum hybrids ('P-82G63', 'RB-Patrón', 'DK-54' and 'Ámbar') to application of two herbicides (prosulfuron and 2,4-D) at two dosages (1X recommended dose: prosulfuron at $22.8 \mathrm{~g}$ i.a. ha $^{-1}$ and 2,4-D at $720 \mathrm{~g}$ i.a. $\mathrm{ha}^{-1}$; and $1.5 \mathrm{X}$ recommended dose), at two phenological stages (V5, 5 expanded leaves; and V8, 8 expanded leaves) was tested in field trials. No significant differences were observed among genotypes in response to herbicide treatments, but there were significant effects of dosages and herbicides. Prosulfuron $1 \mathrm{X}$ applied at V5 caused only a light toxicity of $6 \%$ that reduced crop height. Height reduction was more pronounced $(19 \%)$ when the $1.5 \mathrm{X}$ dosage was applied at V8 stage; however, no yield reduction was observed in any case. 2,4-D applied in plants at $\mathrm{V} 5$ and $\mathrm{V} 8$ at $1 \mathrm{X}$ and $1.5 \mathrm{X}$ caused significant toxicity from 12 to $24 \%$, and reduced grain yield from 12 to $16 \%$, because of a decrease in the number of grains per panicle. Thus, 2,4-D spraying on sorghum at those stages should be avoided. For sorghum, prosulfuron is more suitable than 2,4-D, because it causes less toxicity.
Index words: Sorghum bicolor, herbicide tolerance, plant height, grain yield.

\section{INTRODUCCIÓN}

En México, el sorgo (Sorghum bicolor L. Moench.) ocupa el segundo lugar después del maíz (Zea mays L.), con 1.9 millones de hectáreas sembradas en 2012. Tamaulipas es el estado con mayor producción de sorgo en México. En 2012 en dicho estado se cosecharon 873 mil hectáreas con una producción de 2.8 millones de toneladas, de las cuales la región norte produjo 2.4 millones de toneladas en 649 mil hectáreas en el ciclo otoño-invierno (SAGARPA, 2012).

El control deficiente de malezas es uno los principales factores que limitan la producción de sorgo en el norte de Tamaulipas. En el ciclo otoño-invierno, la maleza que causa más pérdidas en el rendimiento del cultivo es el polocote (Helianthus annuus L.) (Rosales-Robles et al., 2005a). Otras malezas comunes en esta región son el quelite (Amaranthus palmeri S. Wats), la amargosa (Parthenium hysterophorus L.), el trompillo (Solanum elaeagnifolium Cav.) y la oreja de ratón (Convolvulus arvensis L.) (Rosales-Robles et al., 2005b). La competencia que ejercen las malezas puede reducir severamente el rendimiento de sorgo cuando no son controladas eficientemente en las primeras siete semanas de desarrollo del cultivo (Rodrigues et al., 2010; RosalesRobles et al., 2005a).

Para el control de malezas los productores del sorgo de norte de Tamaulipas realizan de dos a tres escardas, que regularmente complementan con la aplicación de herbicidas y en algunos casos con deshierbes manuales. En sorgo, el 2,4-D amina (referido posteriormente como 2,4-D) es el herbicida más usado para el control selectivo de malezas de hoja ancha en post-emergencia (POST), por su bajo costo. Comúnmente este herbicida se aplica en POST cuando las plantas de sorgo han expuesto de tres a seis hojas y así se obtiene buena selectividad, ya que si se usa fuera de esta etapa de desarrollo causa daños al cultivo (Baumann et al., 1999; Tamado y Milberg, 2004). Otro herbicida postemergente utilizado en sorgo es el prosulfuron, herbicida 
de la familia de sulfonilureas que tiene un buen control de polocote y amargosa, y que se caracteriza por su dosis baja de aplicación, vida media corta y volatilidad baja, y que además minimiza las posibilidades de daño a cultivos noobjetivo (Grichar et al., 2000; O' Sullivan y Thomas 2001; Rosales et al., 2005b). Sin embargo, prosulfuron no ejerce un control eficiente de trompillo y oreja de ratón.

En el norte de Tamaulipas, la toxicidad al sorgo es un problema común en la aplicación de 2, 4-D debido a las dosis y épocas de aplicación inapropiadas y que en algunas ocasiones reduce el rendimiento (Rosales-Robles et al., 2011). La selectividad del 2, 4-D en gramíneas ocurre por inactivación en las plantas no-objetivo, principalmente por el mecanismo de conjugación (Devine et al., 1993). Además, la etapa de desarrollo es importante en la selectividad de este herbicida auxínico, ya que las aplicaciones realizadas cuando el sorgo tiene de 6 a 9 hojas causan daños al cultivo y reducen su rendimiento (Dan et al., 2010; Petter et al., 2011).

El prosulfuron también ocasiona fitotoxicidad en sorgo pero con duración temporal, ya que la selectividad de las sulfonilureas en los cultivos tolerantes se basa en su metabolismo y transporte en las primeras horas después de su aplicación (Ma et al., 1997), por lo que algunas veces se presentan daños ligeros (Brown, 1990). En sorgo de 6 hojas la aplicación de prosulfuron causa una toxicidad ligera que reduce la altura del cultivo sin afectar su rendimiento (Rosales-Robles et al., 2011).

El uso de la dosis adecuada de los herbicidas es otro factor importante para obtener un buen control de maleza sin afectar el desarrollo del sorgo. Es común que la calibración inadecuada del equipo de aspersión resulte en una dosis excesiva de los herbicidas. El objetivo de este trabajo fue evaluar la tolerancia de cuatro genotipos de sorgo en dos etapas de desarrollo a dos dosis de aplicación de 2, 4-D y prosulfuron.

\section{MATERIALES Y MÉTODOS}

Los experimentos de campo se establecieron en los ciclos
Otoño-Invierno 2006, 2007 y 2008 en Río Bravo, Tamaulipas, $\left(27^{\circ} 57^{\prime} \mathrm{LN}, 98^{\circ} 10^{\prime} \mathrm{LO}\right)$. El suelo de los lotes experimentales es de textura arcillosa ( $25 \%$ arena, $18 \%$ limo y 57 $\%$ arcilla), con $\mathrm{pH} 7.2$ y un contenido de materia orgánica de $1.1 \%$. La preparación del suelo consistió en un paso de arado, dos pasos de rastra y surcado a $80 \mathrm{~cm}$. En los tres años que duró esta investigación, se fertilizó con la fórmula $120 \mathrm{~N}-40 \mathrm{P}-00 \mathrm{~K}$, y se proporcionó un riego de siembra más dos adicionales a los 40 y $60 \mathrm{~d}$ después de la siembra. Las fechas de siembra y aplicación de herbicidas se presentan en el Cuadro 1.

Los tratamientos se evaluaron mediante un diseño experimental en parcelas divididas con tres repeticiones. La parcela grande consistió de cuatro híbridos de sorgo ('P-82G63', 'RB-Patrón', 'DK-54' y 'Ámbar'), y la parcela chica consistió de nueve tratamientos resultantes de la combinación de dos herbicidas (prosulfuron y 2, 4-D amina), dos dosis de aplicación (dosis de etiqueta, $1 \mathrm{X}$ y sobredosis de $50 \%, 1.5 \mathrm{X}$ ), y dos épocas de aplicación (V5, cinco hojas liguladas; y V8, ocho hojas liguladas), más un testigo sin aplicación.

Las dosis de etiqueta fueron $22.8 \mathrm{~g}$ i.a. $\mathrm{ha}^{-1}$ para prosulfuron y $720 \mathrm{~g}$ i.a. ha ${ }^{-1}$ para 2, 4-D. Estos herbicidas pueden ser aplicados en POST con buena selectividad en sorgo en la etapa de desarrollo de 3 a 6 hojas liguladas (Regehr et al., 2011). La primera aplicación se hizo cuando el sorgo tenía cinco hojas liguladas (V5) para corroborar la selectividad al cultivo, y la aspersión de los herbicidas se hizo sobre la hilera de plantas cultivadas.

La unidad experimental fue de 4 surcos de $15 \mathrm{~m}$ de largo, y la aplicación de los herbicidas se hizo con un aspersor de mochila motorizado (modelo Forza 25, Swissmex Rapid $\mathbb{R}$; México) provisto con boquillas de abanico plano 8004 con una presión de salida de $275 \mathrm{kPa}$ y un volumen de aspersión de $250 \mathrm{~L} \mathrm{ha}^{-1}$. El sorgo se mantuvo libre de malezas en todos los tratamientos mediante dos escardas y dos deshierbes manuales, para evitar la interferencia de maleza en su desarrollo, y con esto garantizar la determinación precisa del efecto fitotóxico de los herbicidas en los híbridos de sorgo.

La toxicidad al sorgo se evaluó visualmente con base en la

Cuadro 1. Fechas de siembra y de aplicación de los herbicidas prosulfuron y 2 , 4-D amina, en dos etapas fenológicas del sorgo. Río Bravo, Tam.

\begin{tabular}{lccc}
\hline \multirow{2}{*}{ Año } & \multirow{2}{*}{ Fecha de siembra } & \multicolumn{2}{c}{ Etapa fenológica } \\
\cline { 3 - 4 } & & V5 & V8 \\
\hline 2006 & $24-\mathrm{feb}$ & $30-\mathrm{mar}$ & $15-\mathrm{abr}$ \\
2007 & $13-\mathrm{feb}$ & $30-\mathrm{mar}$ & $13-\mathrm{abr}$ \\
2008 & $21-\mathrm{feb}$ & $20-\mathrm{mar}$ & 2 -abr \\
\hline
\end{tabular}

${ }^{\dagger}$ V5 = cinco hojas liguladas; V8 = ocho hojas liguladas 
escala propuesta por la Sociedad Europea de Investigación en Maleza (Frans et al., 1986), como se ilustra en el Cuadro 2. Las evaluaciones se hicieron a los 20 y $40 \mathrm{~d}$ después de la aplicación (DDA) de los herbicidas en V5; y a los 10 y 30 DDA en los tratamientos aplicados en V8. En los mismos días se midió la altura de 10 plantas de sorgo al azar en la parcela útil y se obtuvo el promedio. La altura del sorgo se registró como la distancia vertical de la base de la planta a la hoja central del cogollo en la primera fecha, y al ápice de la panícula en la segunda fecha de muestreo. A la cosecha se registraron los componentes de rendimiento: número de panículas en $5 \mathrm{~m}$ de surco, y con ello se calculó el número de panículas por metro cuadrado; longitud de panícula, peso de 1000 granos y número de granos por panícula, medidos en 10 panículas. El rendimiento de grano se obtuvo al cosechar manualmente las panojas de $7 \mathrm{~m}$ en los dos surcos centrales de cada unidad experimental. Las panojas fueron trilladas y el contenido de humedad del grano se ajustó a $14 \%$.

Los datos de las variables evaluadas se sometieron a análisis de varianza y las medias se compararon con la prueba de Tukey $(P \leq 0.05)$. Antes de su análisis, los datos de fitotoxicidad al cultivo fueron transformados a arcoseno de la raíz cuadrada de $\mathrm{X} / 100$, y los de número de panículas por metro cuadrado y granos por panícula a raíz cuadrada de $\mathrm{X}$, para homogenizar sus varianzas (Lentner y Bishop, 1993). En los resultados se presentan los datos no transformados, con las literales de las pruebas de Tukey de los datos transformados.

\section{RESULTADOS Y DISCUSIÓN}

El análisis combinado de los datos indicó que no hubo efecto de híbridos y años, ni de su interacción en las varia- bles evaluadas, por lo que los datos que aquí se presentan son el promedio de los tres años y de los cuatro híbridos evaluados.

\section{Fitotoxicidad}

Prosulfuron causó menor toxicidad al sorgo que el 2,4-D cuando ambos fueron aplicados con sus dosis de etiqueta en la etapa inadecuada de desarrollo (V8). A los 20 y 40 DDA prosulfuron aplicado en V5 a la dosis de etiqueta (22.8 g i.a. $\mathrm{ha}^{-1}$ ) causó daños ligeros en la altura de la planta (Cuadros 2 y 3). La toxicidad al sorgo causada por prosulfuron ya había sido reportada por Rosales-Robles et al. (2005b y 2011).

La selectividad de este herbicida al sorgo se debe a que es metabolizado por la planta en las primeras horas después de su aplicación, y en algunas ocasiones le causa daños (Brown, 1990). La aplicación de prosulfuron a 1.5X en V5 causó daño evidente al cultivo, daño que aumentó al nivel medio al aplicar ambas dosis en V8 (Cuadro 3). Los porcentajes de daño fueron estadísticamente diferentes entre estados de desarrollo. Los resultados obtenidos a los 40 DDA fueron similares a los registrados a los $20 \mathrm{DDA}$, aunque con una ligera disminución en los daños al sorgo. Entre las dosis de prosulfuron se detectaron diferencias significativas en la fitotoxicidad, pero en ningún caso fue mayor al nivel medio. En estudios anteriores se reportaron sólo daños ligeros de prosulfuron aplicado a sorgo en V8 (Gigón et al., 2009).

En general, con ambas dosis y épocas de aplicación el 2,4-D causó toxicidad de nivel evidente a nivel medio (Cuadro 3) al sorgo, caracterizada por curvatura de las plantas y malformación de sus hojas, daños típicos de herbicidas hormonales en tejidos jóvenes de gramíneas (Baumann et

Cuadro 2. Escala propuesta por la Sociedad Europea de Investigación en Maleza (EWRS) para evaluar el control de maleza y
fitotoxicidad al cultivo por herbicidas.
\begin{tabular}{lcccc}
\hline Valor & Control de maleza (\%) & Efecto en la maleza & Fitotoxicidad al cultivo (\%) & Efecto en el cultivo \\
\hline 1 & $99.0-100.0$ & Muerte & $0.0-1.0$ & Sin efecto \\
2 & $96.5-99.0$ & Muy buen control & $1.0-3.5$ & Síntomas muy ligeros \\
3 & $93.0-96.5$ & Buen control & $3.5-7.0$ & Síntomas ligeros \\
4 & $87.5-93.0$ & Control suficiente & $7.0-12.5$ & Síntomas evidentes sin efecto en \\
& & & & rendimiento \\
5 & $80.0-87.5$ & Control medio & $12.5-20.0$ & Daño medio \\
6 & $70.0-80.0$ & Control regular & $20.0-30.0$ & Daño elevado \\
7 & $50.0-70.0$ & Control pobre & $30.0-50.0$ & Daño muy elevado \\
8 & $1.0-50.0$ & Control muy pobre & $50.0-99.0$ & Daño severo \\
9 & $0.0-1.0$ & Sin efecto & $99.0-100.0$ & Muerte \\
\hline
\end{tabular}

Tomado de: Frans et al., 1986 
al., 1999; Brown et al., 2004). Los daños mayores fueron causados con la dosis 1.5X aplicada en la etapa V8. Dan et al. (2010) y Petter et al. (2011) también observaron mayores daños al sorgo al aplicar 2, 4-D en V9 en comparación con V5 y V6. Según Dan et al. (2010), la aplicación de 2, 4-D en un estado avanzado de desarrollo del sorgo causa mayores daños debido al desequilibrio hormonal en la transición de la fase vegetativa a la fase reproductiva del cultivo. A los 40 DDA hubo un incremento en la fitotoxicidad de 3 a $7 \%$, sobre todo al aplicar la dosis más alta en el estado de desarrollo más avanzado (V8).

\section{Altura del sorgo}

A los 20 DDA todas las combinaciones de dosis y épocas de aplicación de prosulfuron redujeron significativamente la altura del sorgo con relación al testigo sin aplicación (Cuadro 4), sobre todo al aplicarlo en V8, con reducciones de 13 y $17 \%$ para las dosis de $1 \mathrm{X}$ y $1.5 \mathrm{X}$, respectivamente. La disminución de la altura es un efecto común de los herbicidas sulfonilureas porque afectan la producción de aminoácidos ramificados y la de nuevos tejidos (Baumann et al., 1999). A los 40 DDA se observó que la altura del sorgo se recuperó pero solamente después aplicar prosulfuron a la dosis $1 \mathrm{X}$ en la etapa V5, ya que en esa fecha presentó una altura similar a la del testigo. La reducción de altura por la aplicación de prosulfuron ya había sido reportada previamente en sorgo (Rosales-Robles et al., 2011) y mijo (Panicum miliaceum) (Uludag et al., 1997).

El 2,4-D aplicado a la dosis $1.5 \mathrm{X}$ en V5 redujo $(\mathrm{P} \leq 0.05)$ la altura del sorgo a los $20 \mathrm{DDA}$, mientras que los demás tratamientos fueron similares al testigo (Cuadro 4). A los 40 DDA, los efectos del 2, 4-D se acentuaron ya que las plantas tenían de 6 a $12 \%$ menos altura que el testigo. Además, las plantas tratadas con 2, 4-D presentaron curvatura y malformación de los tallos, síntomas típicos de herbicidas hormonales (Regehr et al., 2011). Estos resultados concuerdan con los reportados por Petter et al., (2011) quienes observaron una reducción de $8 \%$ en la altura del sorgo tratado con 2 , 4-D a 1005 g i.a. ha ${ }^{-1}$ en la etapa de V9.

\section{Rendimiento de grano}

La fitotoxicidad de nivel ligero a medio causada al sorgo por el prosulfuron en las dosis 1 y $1.5 \mathrm{X}$ aplicadas en las dos etapas de desarrollo no se reflejó en el rendimiento de grano, ya que no se observaron diferencias estadísticas entre los tratamientos y el testigo (Cuadro 4). En contraste, todos los tratamientos de $2,4-\mathrm{D}$ redujeron $(\mathrm{P} \leq 0.05)$ el rendimiento del sorgo con relación con el testigo, sobre todo con la dosis $1.5 \mathrm{X}$ aplicada en V8 que provocó una pérdida de $16 \%$. Lo anterior evidencia los altos niveles de fitotoxicidad causados por 2, 4-D al cultivo de sorgo. Petter et al. (2011) también detectaron una reducción de $20 \%$ en el rendimiento de sorgo al aplicar 2, 4-D a 1005 g i.a. ha ${ }^{-1}$ en la etapa V9; de modo similar Dan et al. (2010) reportaron reducciones de 18 a $20 \%$ al aplicar 2, 4-D a 840 g i.a. ha- en sorgo con cinco (V5) y nueve hojas liguladas (V9).

\section{Componentes de rendimiento}

No se detectaron diferencias significativas en el número de panículas de sorgo por metro cuadrado ni en la longitud de panículas entre los tratamientos evaluados (Cuadro 5). En cambio, en el peso de 1000 granos los tratamientos de 2, 4-D presentaron un mayor peso que los de prosulfuron, debido probablemente a que el $2,4-\mathrm{D}$ redujo significativamente el

Cuadro 3. Fitotoxicidad causada al sorgo por prosulfuron y 2,4-D aplicados en dos dosis y dos etapas fenológicas del cultivo. Río Bravo, Tam. 2006 - 2008.

\begin{tabular}{|c|c|c|c|c|}
\hline \multirow{2}{*}{ Herbicida } & \multirow{2}{*}{ Dosis } & \multirow{2}{*}{ Etapa fenológica } & \multicolumn{2}{|c|}{ Fitotoxicidad (\%) } \\
\hline & & & $20 \mathrm{DDA}^{9}$ & $40 \mathrm{DDA}$ \\
\hline \multirow[t]{4}{*}{ Prosulfuron } & $1 X^{\dagger}$ & $\mathrm{V} 5^{\dagger \dagger}$ & $6 \mathrm{~g}$ & $6 \mathrm{~d}$ \\
\hline & $1 \mathrm{X}$ & V8 & $16 \mathrm{bc}$ & $14 \mathrm{c}$ \\
\hline & $1.5 \mathrm{X}$ & V5 & $9 \mathrm{f}$ & $7 \mathrm{~d}$ \\
\hline & $1.5 \mathrm{X}$ & V8 & $18 \mathrm{ab}$ & $17 \mathrm{bc}$ \\
\hline \multirow[t]{4}{*}{$2,4-\mathrm{D}$} & $1 \mathrm{X}$ & V5 & $12 \mathrm{e}$ & $16 \mathrm{c}$ \\
\hline & $1 \mathrm{X}$ & V8 & $13 \mathrm{de}$ & $20 \mathrm{~b}$ \\
\hline & $1.5 \mathrm{X}$ & V5 & $14 \mathrm{~cd}$ & $19 \mathrm{~b}$ \\
\hline & $1.5 \mathrm{X}$ & V8 & $19 \mathrm{a}$ & $24 \mathrm{a}$ \\
\hline \multicolumn{3}{|c|}{ Testigo sin aplicación } & $0 \mathrm{~h}$ & $0 \mathrm{e}$ \\
\hline
\end{tabular}

${ }^{\dagger}$ Prosulfuron $1 \mathrm{X}=22.8$ y $1.5 \mathrm{X}=34.2 \mathrm{~g}$ i.a.ha ${ }^{-1} ; 2,4-\mathrm{D} 1 \mathrm{X}=720$ y $1.5 \mathrm{X}=1080 \mathrm{~g}$ i.a.ha ${ }^{-1}$; ${ }^{\dagger \dagger} \mathrm{V} 5=$ cinco hojas liguladas; V8 = ocho hojas liguladas; ${ }^{9} \mathrm{DDA}$ = días después de aplicación del herbicida; Medias $(\mathrm{n}=36)$ con letras iguales en cada columna no son estadísticamente diferentes (Tukey, 0.05). 
número de granos por panícula. La reducción del número de granos por panícula se ha señalado como el principal factor en la reducción del rendimiento en sorgo sujeto a diferentes tipos de estrés (Saeed et al., 1986; Craufurd y Peacock, 1993; Rosales-Robles et al., 2005a). Además, el 2,4-D es un herbicida auxínico que aumenta el aborto de flores y reduce el número de granos por panícula en sorgo (Petter et al., 2011). Los tratamientos de prosulfuron no fueron estadísticamente diferentes al testigo en los componentes de rendimiento de grano.

\section{CONCLUSIONES}

Los híbridos de sorgo 'P-82G63, 'RB-Patrón', 'DK-54' y 'Ámbar' no difirieron en su respuesta a los herbicidas pro- sulfuron y 2,4-D aplicados en post-emergencia.

Prosulfuron aplicado a la dosis de etiqueta (1X) en plantas de sorgo en las etapas de cinco (V5) y ocho hojas (V8) causó fitotoxicidad ligera y redujo temporalmente la altura de planta, pero no afectó el rendimiento de grano. La aplicación de prosulfuron a una dosis $50 \%$ mayor (1.5X) cuando las plantas estaban en las etapa V5 y V8, causó fitotoxicidad de nivel evidente a medio y redujo significativamente la altura del sorgo pero sin afectar su rendimiento.

El herbicida 2,4-D aplicado a las dosis 1 y $1.5 \mathrm{X}$ a plantas de sorgo en etapas V5 y V8 causó mayor fitotoxicidad que el prosulfuron y redujo su altura y rendimiento, por lo que se debe evitar su aplicación en estas condiciones.

Cuadro 4. Efecto del prosulfuron y 2,4-D aplicados en dos dosis y dos etapas de fenológicas en la altura y rendimiento de grano de sorgo. Río Bravo, Tam. 2006 - 2008.

\begin{tabular}{|c|c|c|c|c|c|}
\hline \multirow{2}{*}{ Herbicida } & \multirow{2}{*}{ Dosis } & \multirow{2}{*}{ Etapa fenológica } & \multicolumn{2}{|c|}{ Altura $(\mathrm{cm})$} & \multirow{2}{*}{ Rendimiento $\left(\mathrm{kg} \mathrm{ha}^{-1}\right)$} \\
\hline & & & $20 \mathrm{DDA}^{9}$ & $40 \mathrm{DDA}$ & \\
\hline \multirow[t]{4}{*}{ Prosulfuron } & $1 \mathrm{X}^{\dagger}$ & $\mathrm{V} 5^{\dagger \dagger}$ & $79.6 \mathrm{bc}$ & $113.7 \mathrm{ab}$ & $6040 \mathrm{ab}$ \\
\hline & $1 \mathrm{X}$ & V8 & $71.7 \mathrm{~d}$ & $109.4 \mathrm{bcd}$ & $5850 \mathrm{abcd}$ \\
\hline & $1.5 \mathrm{X}$ & V5 & $78.7 \mathrm{c}$ & $112.9 \mathrm{~b}$ & $5910 \mathrm{abc}$ \\
\hline & $1.5 \mathrm{X}$ & V8 & $69.5 \mathrm{~d}$ & $107.4 \mathrm{cde}$ & 5750 abcde \\
\hline \multirow[t]{4}{*}{$2,4-\mathrm{D}$} & $1 \mathrm{X}$ & V5 & $80.5 \mathrm{abc}$ & $105.4 \mathrm{de}$ & 5550 bcde \\
\hline & $1 \mathrm{X}$ & V8 & $83.0 \mathrm{ab}$ & $110.8 \mathrm{bc}$ & $5500 \mathrm{cde}$ \\
\hline & $1.5 \mathrm{X}$ & V5 & $79.3 \mathrm{c}$ & $103.8 \mathrm{e}$ & $5350 \mathrm{de}$ \\
\hline & $1.5 \mathrm{X}$ & V8 & $81.2 \mathrm{abc}$ & $108.0 \mathrm{cde}$ & $5260 \mathrm{e}$ \\
\hline Testigo sin aplicación & & & $83.2 \mathrm{a}$ & $117.7 \mathrm{a}$ & $6240 \mathrm{a}$ \\
\hline
\end{tabular}

${ }^{\dagger}$ Prosulfuron $1 \mathrm{X}=22.8$ y $1.5 \mathrm{X}=34.2 \mathrm{~g}$ i.a.ha ${ }^{-1} ; 2,4-\mathrm{D} 1 \mathrm{X}=720$ y $1.5 \mathrm{X}=1080 \mathrm{~g}$ i.a.ha ${ }^{-1} ;{ }^{\dagger \dagger} \mathrm{V} 5=$ cinco hojas liguladas; $\mathrm{V} 8=0$ ocho hojas liguladas; ${ }^{\circ} \mathrm{DDA}=\mathrm{Días}$ después de aplicación del herbicida; Medias $(\mathrm{n}=36)$ con letras iguales en cada columna no son estadísticamente diferentes (Tukey, 0.05).

Cuadro 5. Efecto del prosulfuron y 2,4-D aplicados en dos dosis y dos etapas fenológicas en los componentes de rendimiento de grano de sorgo. Río Bravo, Tam. 2006 - 2008.

\begin{tabular}{lcccccc}
\hline Herbicida & Dosis & Etapa fenológica & $\begin{array}{c}\text { Panículas } \\
\left(\mathrm{m}^{-2}\right)\end{array}$ & $\begin{array}{c}\text { Longitud de } \\
\text { panícula }(\mathrm{cm})\end{array}$ & $\begin{array}{c}\text { Peso } 1000 \text { granos } \\
(\mathrm{g})\end{array}$ & $\begin{array}{c}\text { Granos por } \\
\text { panícula }\end{array}$ \\
\hline Prosulfuron & $1 \mathrm{X}^{\dagger}$ & $\mathrm{V}^{\dagger \dagger}$ & $16.3 \mathrm{a}$ & $25.3 \mathrm{a}$ & $27.8 \mathrm{~d}$ & $1158 \mathrm{a}$ \\
& $1 \mathrm{X}$ & $\mathrm{V} 8$ & $16.6 \mathrm{a}$ & $25.9 \mathrm{a}$ & $26.8 \mathrm{~d}$ & $1176 \mathrm{a}$ \\
& $1.5 \mathrm{X}$ & $\mathrm{V} 5$ & $16.5 \mathrm{a}$ & $25.9 \mathrm{a}$ & $27.2 \mathrm{~d}$ & $1131 \mathrm{a}$ \\
$2,4-\mathrm{D}$ & $1.5 \mathrm{X}$ & $\mathrm{V} 8$ & $16.6 \mathrm{a}$ & $25.3 \mathrm{a}$ & $26.9 \mathrm{~d}$ & $1151 \mathrm{a}$ \\
& $1 \mathrm{X}$ & $\mathrm{V} 5$ & $15.9 \mathrm{a}$ & $27.1 \mathrm{a}$ & $36.2 \mathrm{a}$ & $870 \mathrm{~b}$ \\
& $1 \mathrm{X}$ & $\mathrm{V} 8$ & $16.3 \mathrm{a}$ & $25.7 \mathrm{a}$ & $32.2 \mathrm{bc}$ & $922 \mathrm{~b}$ \\
& $1.5 \mathrm{X}$ & $\mathrm{V} 5$ & $15.8 \mathrm{a}$ & $26.4 \mathrm{a}$ & $35.7 \mathrm{ab}$ & $864 \mathrm{~b}$ \\
Testigo sin aplicación & $1.5 \mathrm{X}$ & $\mathrm{V} 8$ & $16.3 \mathrm{a}$ & $25.0 \mathrm{a}$ & $34.1 \mathrm{ab}$ & $855 \mathrm{~b}$ \\
\hline
\end{tabular}

${ }^{\dagger}$ Prosulfuron $1 \mathrm{X}=22.8$ y $1.5 \mathrm{X}=34.2 \mathrm{~g}_{\text {i.a.ha }}{ }^{1} ; 2,4-\mathrm{D} 1 \mathrm{X}=720$ y $1.5 \mathrm{X}=1080 \mathrm{~g}$ i.a.ha ${ }^{-1} ;{ }^{\dagger \dagger} \mathrm{V} 5=$ cinco hojas liguladas; $\mathrm{V} 8=$ ocho hojas liguladas; Medias $(\mathrm{n}=36)$ con letras iguales en cada columna no son estadísticamente diferentes (Tukey, 0.05). 


\section{AGRADECIMIENTOS}

A la Fundación Produce Tamaulipas, A.C. por el apoyo financiero para realizar este trabajo, y a Esteban Robles Escarriola por su apoyo técnico en la conducción de la investigación.

\section{BIBLIOGRAFÍA}

Baumann P. A., P. A. Dotray and E. P. Prostko (1999) Herbicides: how they work and the symptoms they cause. Texas Agricultural Extension Service. Texas A\&M University. B-6081. Texas, USA. $12 \mathrm{p}$.

Brown H. M. (1990) Mode of action, crop selectivity, and soil relations of the sulfonylurea. Pesticide Science 29:263-281.

Brown D. W., K. Al-Khatib, D. L. Regher, P. W. Stahlman and T. M. Loughin (2004) Safening grain sorghum injury from metsulfuron with growth regulator herbicides. Weed Science 52:319. 325.

Craufurd P. Q. and J. M. Peacock (1993) Effect of heat and drought stress on sorghum. II Grain yield. Experimental Agriculture 29:77-86.

Dan H. A., L. G. M. Dan, A. L. L. Barroso, R. S. Oliveira Jr., N. Guerra and C. Feldkircher (2010) Tolerância do sorgo granífero ao 2,4-D aplicado em pós-emergência. Planta Daninha 28:785792.

Devine M. D., S. O. Duke and C. Fedtke (1993) Physiology of Herbicide Action. Prentice Hall, Inc. New Jersey, USA. 441 p.

Frans R., R. Talbert, D. Marx and H. Crowley (1986) Experimental design and techniques for measuring and analyzing plant response to weed control practices. In: Research Methods in Weed Science. $3^{\text {rd }}$ ed. N D Camper (ed). Southern Weed Science Society, Champaign, IL. USA. pp:20-46.

Gigón R., M. R. Vigna y R. L. López (2009) Control químico postemergente de Polygonum convolvulus y Diplotaxis tenuifolia en dos estados fenológicos del cultivo de sorgo granífero. Boletín Informativo. INTA. Buenos Aires, Argentina. 4 p.

Grichar W. J., D. C. Sestak, K. Brewer and B. Minton (2000) Weed control with CGA 152005 and peanut (Arachis hypogaea) response. Weed Technology 14:218-222.
Lentner M. and T. Bishop (1993) Experimental Design and Analysis. Valley Book Co. Virginia, USA. 585 p.

Ma G., H. D. Coble, F. T. Corbin and J. D. Burton (1997) Physiological mechanisms for differential responses of three weed species to prosulfuron. Weed Science 45:642-647.

O'Sullivan J. and R. J. Thomas (2001) Injury and yield effects of crops grown in CGA-152005 treated soil. Weed Technology 15:594597.

Petter F. A., L. P. Pacheco, F. Alcántara-Neto, A. M. Zuffo, S. O. Procopio and F. A. Almeida (2011) Desempenho agronômico do sorgo em função de doses e épocas de aplicação do herbicida 2,4-D. Planta Daninha 29:1091-1098.

Regehr D. L., D. E. Peterson, W. H. Fick, P. W. Stahlman and R. E. Wolf (2011) Chemical Weed Control for Field Crops, Pastures, Rangeland, and Noncropland. Report of Progress 1045. Kansas State University. Kansas, USA. 123 p.

Rodrigues A. C. P., N. V. Costa, L. A. Cardoso, C. F. Campos and D. Martins (2010) Períodos de interferência de plantas daninhas na cultura do sorgo. Planta Daninha 28:23-31.

Rosales-Robles E., R. Sanchez-de-la-Cruz y J. Salinas-García (2005a) Período crítico de competencia del polocote (Helianthus annuus) en sorgo para grano. Agrociencia 39:205-210.

Rosales-Robles E., R. Sanchez-de-la-Cruz, J. Salinas-García and V. Pecina-Quintero (2005b) Broadleaf weed management in grain sorghum with reduced rates of postemergence herbicides. Weed Technology 19:385-290.

Rosales-Robles E., R. Sanchez-de-la-Cruz y P. A. Cerda-García (2011) Control químico de maleza de hoja ancha en sorgo para grano. Revista Fitotecnia Mexicana 34:269-275.

Saeed M., C. A. Francis and M. D. Clegg (1986) Yield component analysis in grain sorghum. Crop Science 26:346-351.

SAGARPA, Secretaría de Agricultura, Ganadería, Desarrollo Rural, Pesca y Alimentación (2012). SIAP. Sistema de Información Agroalimentaria y Pesquera. Disponible en: http://www.siap. sagarpa.gob.mx/ (Octubre 2012).

Tamado T. and P. Milberg (2004) Control of parthenium (Parthenium hysterophorus) in grain sorghum (Sorghum bicolor) in the smallholder farming system in eastern Ethiopia. Weed Technology 18:100-105.

Uludag A., D. J. Lyon, S. J. Nissen and S. D. Kachman (1997) Proso millet (Panicum miliaceum) response to CGA-152005 (prosulfuron). Weed Technology 11:138-143. 